\title{
Estudio de la degradación y ruptura de estructuras MOS basadas en óxidos high- $k$ multicapas
}

\section{Study of the ware out and breakdown of MOS structures based on multilayered high- $k$ oxides}

Presentación: 6-7/10/2020

\section{Doctorando:}

\section{Santiago Boyeras Baldomá}

Laboratorio de Nanoelectrónica, Unidad de Investigación y Desarrollo de las Ingenierías, Facultad Regional Buenos Aires, Universidad Tecnológica Nacional - Argentina

sboyeras@frba.utn.edu.ar

\section{Director:}

\section{Félix Palumbo}

\section{Resumen}

El crecimiento exponencial de la industria electrónica ha sido impulsado por un aumento en la densidad de transistores complementarios metal-óxido-semiconductor (CMOS). Pero la tecnología de transistores basada en Silicio está cerca de los límites físicos de la miniaturización, amenazando con acabar con la revolución microelectrónica. Actualmente, se están realizando importantes avances tecnológicos en cuanto a una nueva generación de dispositivos basados en una combinación inteligente de materiales seleccionados. En este trabajo se estudia el desgaste y ruptura de dispositivos MOS basados en óxidos multicapa de alta permitividad. Los resultados experimentales sugieren que el uso de óxidos con alta conductividad térmica tiene un fuerte impacto en la dinámica de ruptura de los dispositivos.

Palabras clave: MOS, fiabilidad, ruptura, multicapas.

\begin{abstract}
The exponential growth of the electronics industry has been driven by an increase in the density of complementary metal-oxidesemiconductor (CMOS) transistors. But silicon-based transistor technology is close to the physical limits of miniaturization, threatening to end the microelectronics revolution. Currently, important technological advances are being made in terms of a new generation of devices based on an intelligent combination of selected materials. In this work, the wear out and breakdown of MOS devices based on multilayered high- $k$ oxides is studied. The experimental results suggest that the use of oxides with a high thermal conductivity has a strong impact on the breakdown dynamics of the devices.
\end{abstract}

Keywords: MOS, reliability, breakdown, multilayers.

\section{Introducción}

La revolución de la microelectrónica podría caracterizarse por el atributo único del transistor de efecto de campo MOSFET (metal-oxide-semiconductor field-effect transistor) que mejora sus características a medida que se reducen sus dimensiones (Ferain, 2011). Debido a que los MOSFET han disminuido en tamaño siguiendo una ley geométrica, la velocidad de conmutación y su densidad en un circuito integrado han aumentado exponencialmente, mientras que la energía de conmutación 

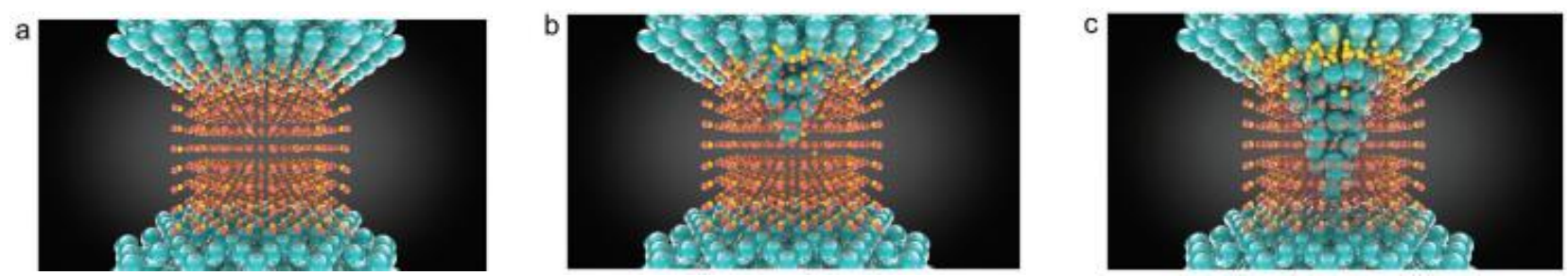

Figura 1: Esquema de la formación de un filamento conductor en pilas MIM (metal/insulator/metal). La penetración de iones metálicos desde el electrodo siempre va acompañada de una severa generación de vacantes. La formación del camino percolativo finaliza cuando un filamento conductor une ambos electrodos y se produce la falla del dispositivo.

ha disminuido de forma similar (Iwai, 2009). Estas características de la miniaturización del MOSFET han impulsado la revolución de la micro- y nanoelectrónica.

Recientemente, la miniaturización del MOSFET entró en una fase donde la densidad de potencia disipada por los circuitos integrados lógicos alcanza aproximadamente los $100 \mathrm{~W} / \mathrm{cm}^{2}$ (Frank, 2002). La densidad de potencia no puede aumentar mucho más sin modificar el encapsulado y costos de enfriamiento, lo que generaría que estos integrados (chips) sean poco prácticos para la mayoría de las aplicaciones. El progreso continuo en la densidad de transistores en los "chips" requeriría una reducción en la tensión de operación (Iwai, 2009; Frank, 2002), pero esto comprometería la velocidad de conmutación. Por lo tanto, la miniaturización del transistor MOSFET necesita innovaciones para eludir las barreras de los mecanismos físicos que limitan al dispositivo. Las opciones tecnológicas para mejorar esos aspectos generalmente se dividen en dos categorías: nuevos materiales y nuevas estructuras de dispositivos (Buchanan, 1999; Wong, 2002).

Es en este contexto, donde el estudio de la fiabilidad aparece como un campo de gran importancia. Para poder proyectar estas tecnologías a la industria y, de esta forma, continuar con la revolución electrónica, es vital el conocimiento sobre los mecanismos de ruptura. Para dispositivos MOS en funcionamiento nominal (un régimen de bajo voltaje y campos eléctricos altos), el proceso de desgaste del dieléctrico conduce a la llamada ruptura dieléctrica dependiente del tiempo (TDBD, time-dependent breakdown) (Lombardo et al., 2005). Tal fenómeno da como resultado una pérdida de rendimiento o incluso la falla catastrófica del dispositivo. Por lo tanto, para hacer predicciones confiables sobre la vida útil del dispositivo que cumplan con los estándares de la industria, la dinámica de ruptura debe ser bien entendida.

Estudios recientes (Palumbo, Lombardo, Eizenberg, 2014) han confirmado que el evento de ruptura comienza cuando se completa la formación de un camino de percolación que une el ánodo y el cátodo cuando una estructura MOS o MIM está sometida a una diferencia de potencial (Figura 1). Se trata de una ruta de defectos puntuales en el óxido que desencadena el transporte de electrones a través del óxido. El evento de ruptura en dieléctricos tradicionales con un espesor menor a $20 \mathrm{~nm}$ se caracteriza por una pérdida progresiva de sus propiedades aislantes, en lugar de un cambio brusco en el mecanismo de conducción observado en capas dieléctricas gruesas (>50 nm) (Palumbo et al., 2020). Este fenómeno, a menudo denominado ruptura progresiva, puede reconocerse por un aumento prolongado de la corriente a través del dieléctrico hasta alcanzar un nivel prohibitivo para las condiciones de operación de los MOSFET.

La ruptura progresiva se debe a la transferencia de energía del camino percolativo a su entorno, lo que promueve la electro-migración de las especies atómicas más rápidas entre las disponibles, proporcionando la construcción del filamento de ruptura. En nuestros trabajos recientes, se estudia la ruptura progresiva de estructuras innovadoras basadas en los siguientes óxidos: Óxido de Aluminio $\left(\mathrm{Al}_{2} \mathrm{O}_{3}\right)$, conocido por ser un óxido de barrera alta, así como también por tener una alta conductividad térmica; y Óxido de Hafnio, famoso por su alta constante dieléctrica. Los resultados sugieren que la presencia de un óxido con alta conductividad térmica tiene un fuerte impacto en la dinámica de ruptura del dispositivo, disminuyendo el parámetro que la caracteriza $\left(d I_{B D} / d t\right)$; y que la disipación de calor en las estructuras no se ve afectada por la presencia de interfaces entre óxidos, siendo ésta dominada por los materiales bulk.

\section{Desarrollo}

Los dispositivos bajo estudio se dividen en dos grupos. Por un lado, dispositivos MOS con un óxido de espesor de 100 $\AA$. Este espesor se divide entre $\mathrm{Al}_{2} \mathrm{O}_{3}$ y HfO 2 de distintas formas: 0-100, 5-95, 10-90, 30-70, 35-65, 40-60, 100-0 (en $\AA$ ). De esta forma, estudiamos dispositivos hechos íntegramente de $\mathrm{Al}_{2} \mathrm{O}_{3}$, otros de $\mathrm{HfO}_{2}$, y otros con combinaciones de los dos óxidos (ver 


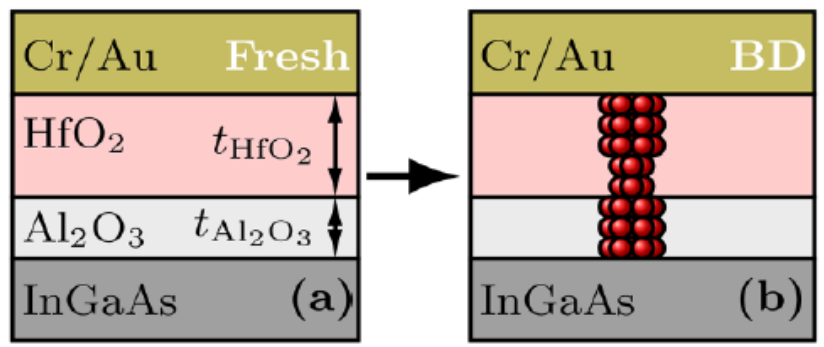

Sample A

Ni contact $(200 \mathrm{~nm})$

$\mathrm{Al}_{2} \mathrm{O}_{3}(10 \mathrm{~nm})$

$\mathrm{HfO}_{2}(10 \mathrm{~nm})$

n-Silicon
Sample B

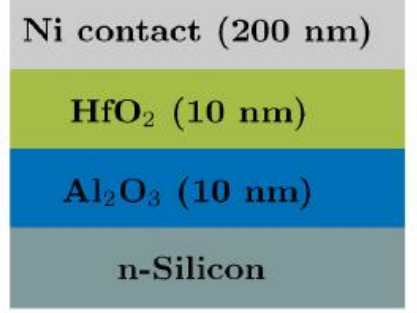

Figura 2: (a) Estructura del primer grupo de dispositivos utilizados. El espesor total del capacitor es de $10 \mathrm{~nm}$, distribuido de distintas formas entre $\mathrm{Al}_{2} \mathrm{O}_{3}$ y $\mathrm{HfO}_{2}$. (b) Representación del camino percolativo formado a través del óxido bicapa. En esta instancia, ambos electrodos se encuentran unidos por un filamento conductivo: el dispositivo se encuentra roto.

Figura 3: Para el segundo grupo de dispositivos, el óxido de varias capas es compuesto por combinaciones de $\mathrm{HfO}_{2}$ y $\mathrm{Al}_{2} \mathrm{O}_{3}$. $\mathrm{El}$ espesor total del óxido permanece constante para todas las muestras: $20 \mathrm{~nm}$. La figura no está a escala.

Figura 2). Estos óxidos fueron crecidos sobre sustrato InGaAs, y tienen por encima un contacto de $\mathrm{Cr} / \mathrm{Au}$. La idea detrás de esta estructura es combinar distintos óxidos para beneficiarse de las propiedades de cada uno. Por otro lado, el segundo grupo de dispositivos posee un espesor total de $200 \AA$, y se encuentran conformados por dos o tres capas de óxidos, bajo distintas combinaciones, como se ilustra en la Figura 3.

Para estudiar la ruptura, se realizan experimentos de estrés a tensión constante (constant-voltaje stress, CVS). Estos experimentos son llevados a cabo en el Laboratorio de Nanoelectrónica de la UTN.BA. Se somete el dispositivo a una tensión (que suele ser mayor a la tensión de trabajo en condiciones normales para acelerar, de esta forma, la degradación del dispositivo), y se registra la corriente a través del óxido. Los transientes de corriente-tiempo se obtienen utilizando dos configuraciones diferentes. Con un Source-Measurement Unit (SMU) de Keithley, nos aseguramos de capturar la evolución completa del transiente actual, registrando corrientes menores al $n A$, hasta valores límites de unos pocos $m A$. La Figura 4 (a) ilustra transientes obtenidos utilizando este setup para la muestra D (3 capas) utilizando distintas tensiones. Para una mejor resolución (hasta unos pocos microsegundos) capturamos una parte del transiente de corriente usando un amplificador de transimpedancia de alto ancho de

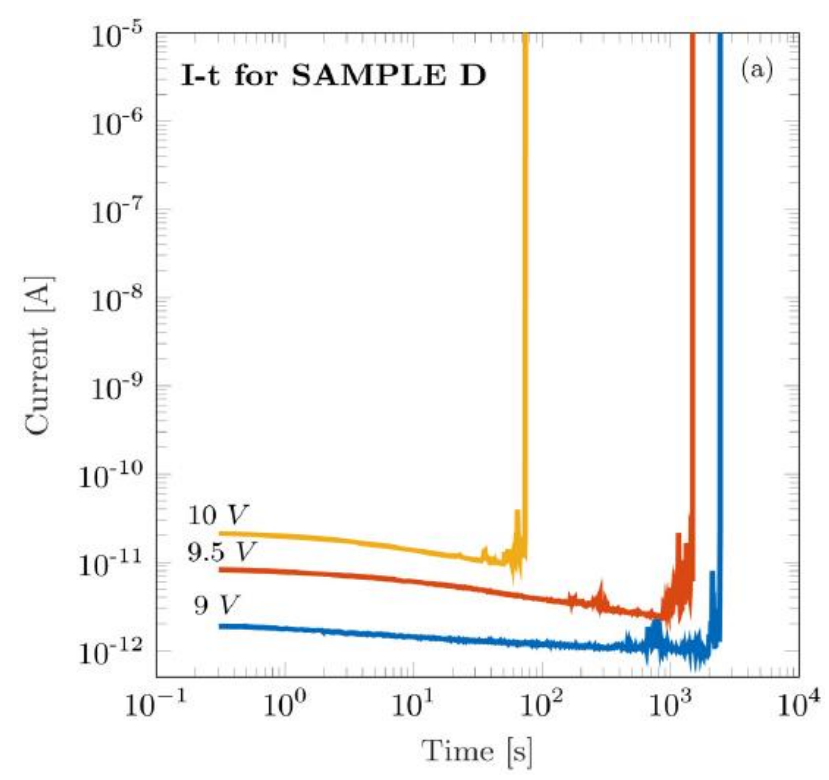

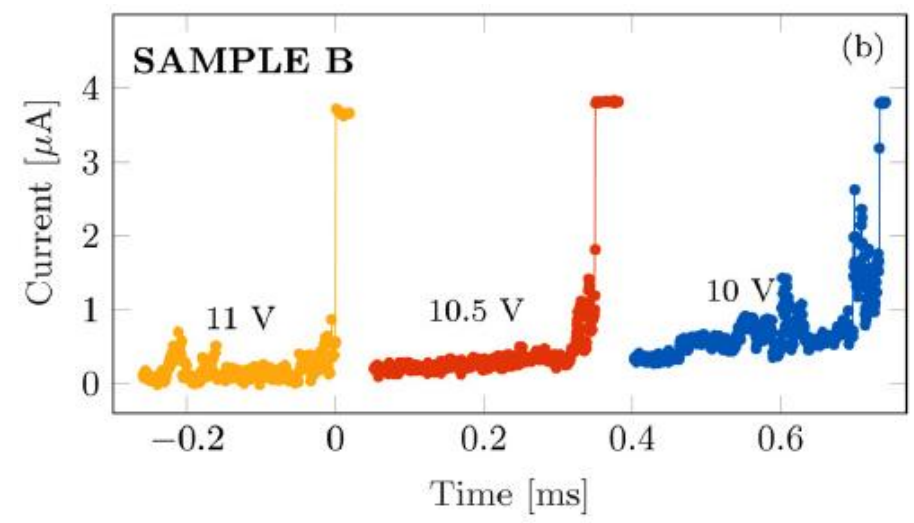

Figura 4: (a) Transientes obtenidos utilizando el SMU de Keithley sobre la muestra B. (b) Transientes obtenidos utilizando el setup de mayor resolución temporal. 
banda (TIA) y un osciloscopio de muestreo digital (DSO) para alimentar el dispositivo y registrar la corriente amplificada. Transientes obtenidos de esta forma se ilustran en la Figura 4 (b), sobre la muestra B (dos capas) con distintas tensiones.

Figura 5: Transitorio de ruptura $(\mathrm{I}-\mathrm{t})$ adquirido utilizando SMU para los dispositivos de $100 \AA \mathrm{HfO}_{2}$. El recuadro muestra un transiente I-t capturada por medio de un TIA con una ganancia de $10^{5} \mathrm{~V} / \mathrm{A}$.

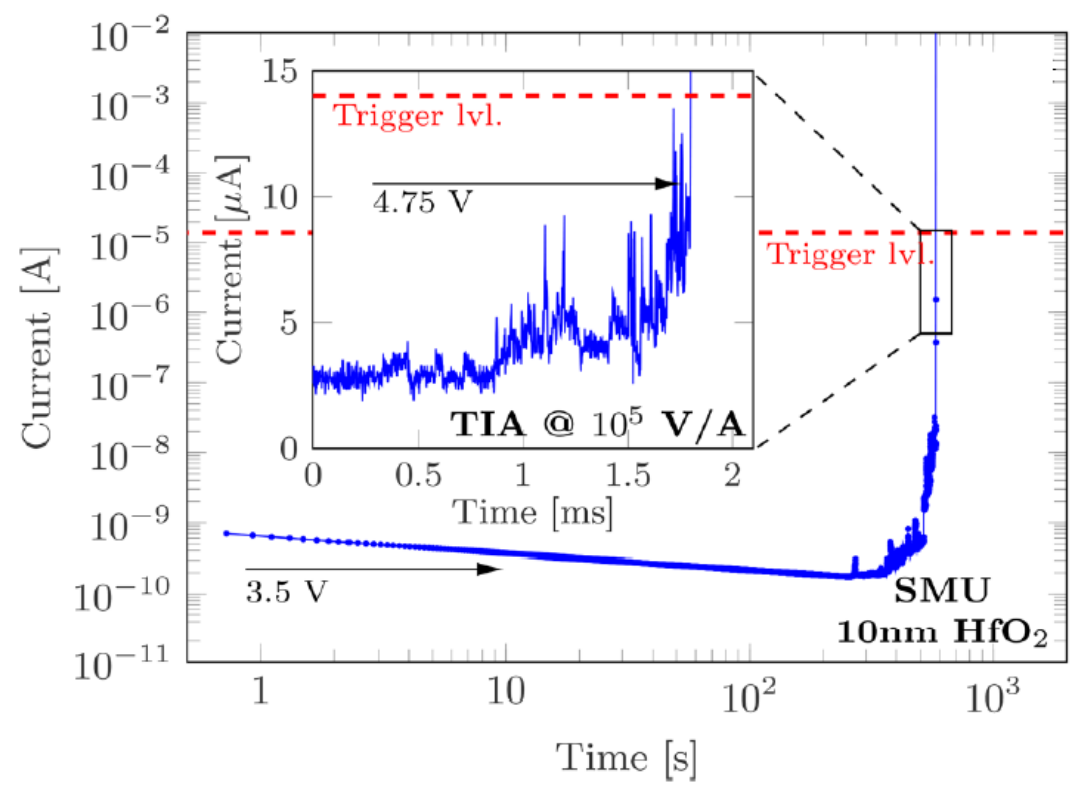

Los datos experimentales se procesan para caracterizar el dispositivo en su forma original y la dinámica del desgaste y la posterior ruptura. La Figura 5 muestra los resultados obtenidos utilizando los dos setups superpuestos en el mismo gráfico. Todos los transientes de ruptura obtenidos tienen una forma similar: al comienzo, la corriente se mantiene constante, o decrece levemente. Luego, se produce un cambio en esta tendencia, comenzando a crecer el valor de corriente de una forma ruidosa: a partir de aquí, el dispositivo está roto. Sin embargo, en los transientes vemos que, a pesar de estar roto, el dispositivo es capaz de mantener el mismo nivel de corriente por un periodo de tiempo, hasta que finalmente el valor de la corriente salta abruptamente hasta un nuevo valor, órdenes de magnitud mayor: el dispositivo ha fallado. Se llama tasa de degradación (en inglés, degradation rate, DR) al valor de la pendiente de la corriente entre el tiempo de ruptura y el tiempo de falla. A través de este parámetro, se caracteriza la dinámica de ruptura de cada dispositivo $\left(d I_{B D} / d t\right)$. Si se logra reducir el valor de este parámetro, la vida útil del dispositivo se puede incrementar considerablemente (Palumbo, Lombardo, Eizenberg, 2014).

\section{Impacto del espesor relativo de óxidos en la ruptura de dispositivos bicapa}

Para el primer grupo de dispositivos, se repitieron los experimentos para distintos valores de tensión aplicada. La Figura 6 muestra los resultados para todos los dispositivos de este grupo.

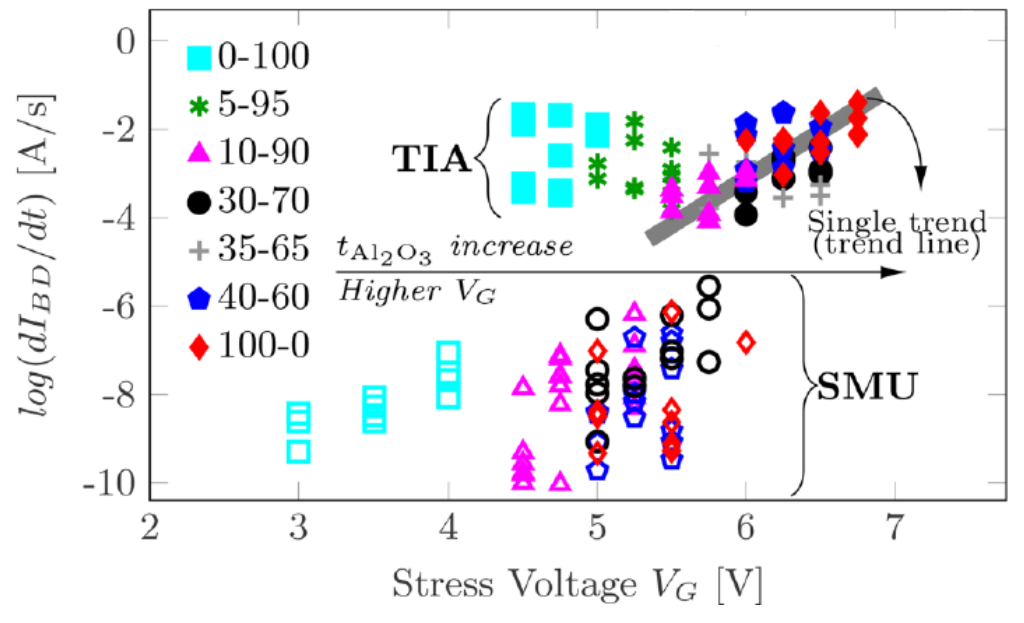

Figura 6: Gráfico de las tasas de degradación extraídas de utilizando los dos tipos de mediciones, SMU y TIA. El valor de la tasa de degradación aumenta con el voltaje aplicado. Se puede observar que al aumentar el espesor relativo de $\mathrm{Al}_{2} \mathrm{O}_{3}$, el voltaje necesario para alcanzar un nivel de tasa de degradación aumenta. 
Mediante la determinación de la tasa de crecimiento de la corriente de ruptura ( $\left.\mathrm{dI} \mathrm{BD}_{\mathrm{BD}} / \mathrm{dt}\right)$, los resultados se compararon en el marco de un modelo de ruptura progresiva basado en electro-migración, originalmente derivado para óxidos de una sola capa (Palumbo, Lombardo, Eizenberg, 2014). Los resultados experimentales muestran que a medida que la capa interfacial de $\mathrm{Al}_{2} \mathrm{O}_{3}$ es más gruesa, se observa un claro aumento en el voltaje aplicado requerido para obtener valores de $\mathrm{dI} \mathrm{BD}_{\mathrm{BD}} / \mathrm{dt}$ en el mismo rango. Sin embargo, este efecto no se observa para espesores superiores a $1 \mathrm{~nm}$ para la capa de $\mathrm{Al}_{2} \mathrm{O}_{3}$. Esto está relacionado tanto con la distribución de tensión eléctrica a través de la estructura bicapa como con las características térmicas de $\mathrm{Al}_{2} \mathrm{O}_{3}$ que contribuyen a reducir la temperatura del punto de ruptura. Una versión primitiva de estos resultados fue presentada en el congreso internacional SB Micro, llevado a cabo en 2019 en Brasil. El trabajo fue reconocido como el mejor trabajo presentado del congreso (best paper award) (Boyeras et al., 2019). Una versión extendida de este trabajo fue publicada recientemente en la revista Journal of Applied Physics (Pazos et al., 2020).

\section{Impacto de las interfaces entre óxidos en la ruptura de dispositivos multicapa}

Para el segundo grupo de dispositivos, la Figura 7 muestra la tasa de degradación graficada para las distintas muestras de la Figura 3. Dichos valores fueron obtenidos de los experimentos llevados a cabo con el setup basado en el amplificador TIA, ya que los transientes resultaron ser muy rápidos para la resolución temporal del SMU. Cada muestra se estresó en un único valor de tensión de 10.5 V. Aunque los resultados presentan una gran dispersión (como se espera, por resultados anteriores informados en la literatura (Palumbo, Lombardo, Eizenberg, 2014; Lombardo, Wu, Stathis, 2017)), no se observa una diferencia significativa en el valor medio encontrado entre las diferentes muestras.

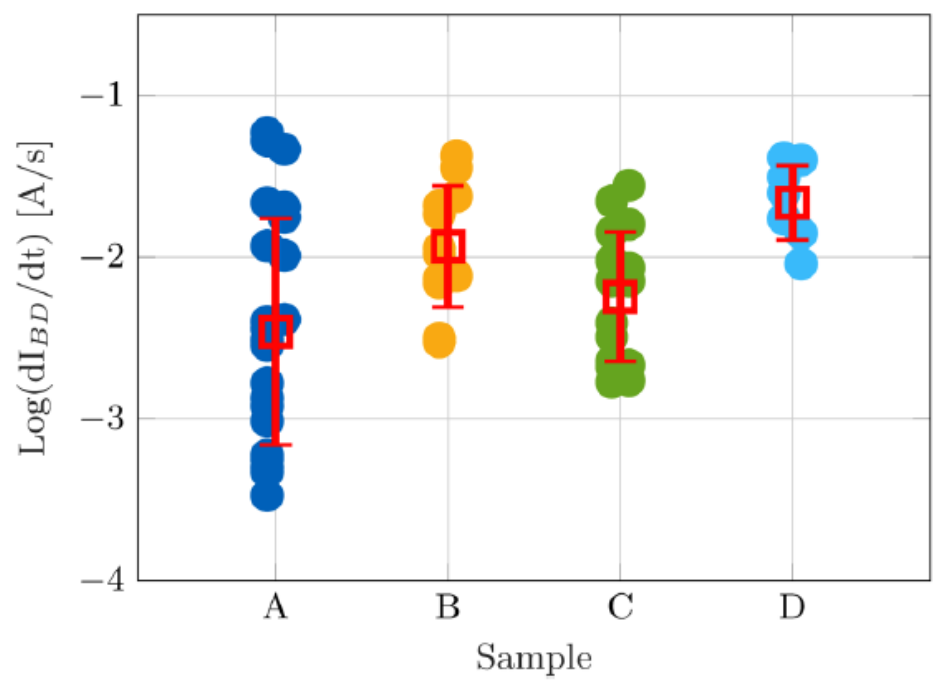

Figura 7: Valores de la tasa de degradación obtenidos para cada muestra multicapa. Se incluyen voltajes positivos como también negativos. Los valores medios obtenidos son similares para todas las muestras, aunque presentan una gran dispersión.

Los resultados experimentales obtenidos sugieren que el número de interfaces óxido-óxido presentes en las estructuras multicapas no tiene un impacto visible en la tasa de crecimiento de la corriente de ruptura para estas muestras. Esto respalda firmemente la interpretación de que los materiales bulk dominan la transferencia de calor en los alrededores de un filamento conductor completamente formado, que no muestra diferencias eléctricas entre las diversas configuraciones de estructuras multicapa. Estos resultados han sido publicados en la revista Journal of Applied Physics (Boyeras Baldomá, Pazos, Aguirre, Palumbo, 2020).

\section{Conclusiones}

Se realizaron estudios experimentales sobre la degradación y ruptura de dispositivos MOS basados en distintas combinaciones de óxidos, logrando caracterizar la dinámica de ruptura a través de la tasa de crecimiento de la corriente de ruptura. Los resultados sugieren que, si en la estructura multicapa se incluye una capa de $\mathrm{Al}_{2} \mathrm{O}_{3}$, disminuye la tasa de degradación para un dado voltaje. Muy posiblemente esto se deba a la alta conductividad térmica del $\mathrm{Al}_{2} \mathrm{O}_{3}$, que permite disipar el calor generado en el punto de ruptura hacia los alrededores con mayor rapidez. Sin embargo, esta mejora llega a un límite cuando la capa de $\mathrm{Al}_{2} \mathrm{O}_{3}$ alcanza los $5 \mathrm{~nm}$. Por otro lado, los resultados experimentales sobre dispositivos MOS multicapa, sugieren que las interfaces óxido-óxido no 
tienen influencia alguna en la dinámica de ruptura de estos dispositivos. Son las propiedades térmicas de los materiales bulk quienes dominan la transferencia de calor.

\section{Agradecimientos}

Estos trabajos recibieron financiamiento de las siguientes instituciones: UTN.BA, bajo los proyectos CCUTIBA5219TC, CCUTIBA4764TC, CCUTNBA0005182, MATUNBA4936; CONICET, bajo los proyectos PIP11220130100077CO y MINCyT bajo los proyectos PICT2016/0579 y PICT2017/2526. Agradecimiento a Prof. Emeritus Moshe Eizenberg y a Igor Krylov, del Department of Materials Science and Engineering Technion, Israel, por proporcionar las muestras bicapa, y a Francesca Campabadal, del Centro Nacional de Microelectrónica, Consejo Superior de Investigaciones Cientificas (IMB-CNM), Spain, por proporcionar las muestras multicapa. Finalmente, agradecimiento especial a mis compañeros que componen el Laboratorio de Nanoelectrónica y que también han hecho su aporte: Ing. Sebastián Pazos, Ing. Fernando Aguirre, Ing. Gabriel Maroli y Dr. Hernan Giannetta.

\section{Referencias}

Boyeras Baldomá, S., Pazos, S. M., Aguirre, F. L., Palumbo, F. (2020). Breakdown transients in high-k multilayered MOS stacks: Role of the oxide-oxide thermal boundary resistance. Journal of Applied Physics 128, 034103. https://doi.org/10.1063/5.0012918

Boyeras, S., Pazos, S. M., Aguirre, F. L., Giannetta, H., Delgado, C., Palumbo, F. (2019). Progressive Breakdown on Bi-Layered Gate Oxide Stacks, 34th Symposium on Microelectronics Technology and Devices (SBMicro), Sao Paulo, Brazil, 2019, pp. 1-4, https://doi.org/10.1109/SBMicro.2019.8919480.

Buchanan, D. A. (1999). Scaling the gate dielectric: Materials, integration, and reliability. IBM J. Res. Dev., vol. 43, no. 3, pp. 245 -264, 1999. https://doi.org/10.1147/rd.433.0245

Ferain, I., Colinge, C. A., Colinge, J. P. (2011). Multigate transistors as the future of classical metal-oxide-semiconductor fieldeffect transistors, Nature 479, 310-316. https://doi.org/10.1038/nature10676

Frank, D. J. (2002). Power-constrained CMOS scaling limits. IBM J. Res. Dev. 46,235-244. https://doi.org/10.1147/rd.462.0235

Iwai, H. (2009). Roadmap for $22 \mathrm{~nm}$ and beyond. Microelectron. Eng. 86, 1520-1528. https://doi.org/10.1016/j.mee.2009.03.129

Lombardo, S., Stathis, J. H., Linder, B. P., Pey, K. L., Palumbo, F. Tung, C. H. (2005). Dielectric breakdown mechanisms in gate oxides. Journal of Applied Physics, 98, 121301. https://doi.org/10.1063/1.2147714

Lombardo, S., Wu, E. Y., Stathis, J. H. (2017). Electron energy dissipation model of gate dielectric progressive breakdown in nand p-channel field effect transistors. J. Appl. Phys. 122, 085701. https://doi.org/10.1063/1.4985794

Palumbo, F., Lombardo, S., Eizenberg, M. (2014). Physical mechanism of progressive breakdown in gate oxides. J. Appl. Phys. 115, 224101. https://doi.org/10.1063/1.4882116

Palumbo, F., Wen, C., Lombardo, S., Pazos, S., Aguirre, F., Eizenberg, M., Hui, F., Lanza, M. (2020). A Review on Dielectric Breakdown in Thin Dielectrics: Silicon Dioxide, High-k, and Layered Dielectrics. Adv. Funct. Mater. 30, 1900657. https://doi.org/10.1002/adfm.201900657

Pazos, S. M., Boyeras Baldomá, S., Aguirre, F. L., Krylov, I., Eizenberg, M., Palumbo, F. (2020). Impact of bilayered oxide stacks on the breakdown transients of metal-oxide-semiconductor devices: An experimental study. Journal of Applied Physics 127, 174101 (2020). https://doi.org/10.1063/1.5138922

Wong, H. S. P. (2002). Beyond the conventional transistor. IBM J. Res. Dev., vol. 46, no. 2/3, pp. 133 -168, 2002. https://doi.org/10.1147/rd.462.0133 